\title{
Using a Mobile Application to Assess Knee Valgus in Healthy and Post-Anterior Cruciate Ligament Reconstruction Participants
}

\author{
Kai-Yu Ho, Brenda Benson Deaver, Tyrel Nelson, and Catherine Turner
}

\begin{abstract}
Context: Popularity of using handheld devices in clinical settings has increased, especially the use of motion analysis applications (MAAs). Video-based measurement tools have been found reliable in measuring knee valgus in subjects without anterior cruciate ligament (ACL) injury. However, there is a need for validation of using a MAA to measure knee valgus in an injured population, given that they may exhibit higher degrees of knee valgus. Objective: To examine the reliability and validity of using a MAA to measure knee valgus during functional activities used to assess return to sport after ACL reconstruction (ACLR). Design: Reliability and validity study. Setting: University laboratory. Participants: Twelve participants with ACLR and 20 healthy individuals. Interventions: Each subject performed single-leg drop landing, single-leg hop, and $90^{\circ}$ cut with simultaneous 3-dimensional (3D) motion capture and video recording on an iPad. Main Outcome Measures: Peak knee valgus during the landing phase was measured using a MAA and 3D analysis. To obtain reliability, peak knee valgus was measured on 2 separate days. Reliability was determined using intraclass correlation coefficients and standard errors of measurement. Validity was assessed using Pearson correlation coefficients by comparing peak knee valgus between the MAA and 3D analysis. The $t$ tests were used to compare knee valgus obtained between raters, within raters, and between the MAA and 3D analysis. Results: Our data revealed excellent intrarater and interrater reliability with low standard errors of measurement of using a MAA for both groups. Significant, moderate to large associations were found in comparing peak knee valgus between the MAA and 3D analysis. However, knee valgus was significantly different between the MAA and 3D analysis across all tasks in both groups. Conclusion: Although a MAA is reliable for measuring peak knee valgus in individuals with ACLR and healthy controls, the actual values obtained by a MAA should be viewed with caution.
\end{abstract}

Keywords: reliability, validity, movement analysis application

Single-leg landing, single-leg hopping, and cutting tests have been widely used in evaluating knee function after anterior cruciate ligament reconstruction (ACLR). ${ }^{1,2}$ It has also been found that females with ACLR showed greater knee valgus, ${ }^{2}$ thereby predisposing them to increased risk of ACL reinjuries. Due to the kinematic deviations following ACLR, it is critical to identify those deficits in clinical practice. Three-dimensional (3D) motion analysis is considered most accurate for evaluating kinematics during dynamic movements; however, it requires expensive equipment, designated space, and extensive training. Two-dimensional (2D) video analysis is more readily available, inexpensive, and is a reliable measure of dynamic knee valgus during various functional tasks. ${ }^{3-6}$ In addition, the existing literature reveals moderate to excellent agreement between the 3D and 2D methods. ${ }^{3-5,7-9}$ However, these comparisons have all been done in individuals without ACL injury/surgery. In addition, although the popularity of motion analysis application (MAA) has increased due to the cheaper cost and easy accessibility of handheld devices, only 1 study was tested with the use of a tablet device ${ }^{8}$ that typically has a lower camera sampling frequency than other computer-based 2D motion analysis.

As individuals with ACLR may exhibit more knee valgus ${ }^{2}$ and there is limited data regarding the reliability/validity of using MAA to measure knee movement, it is necessary for validation of using a MAA to measure knee valgus in individuals with ACLR. Therefore, the purpose of this study was to examine the reliability and

The authors are with the Department of Physical Therapy, University of Nevada, Las Vegas, Las Vegas, NV, USA. Ho (kaiyu.ho@unlv.edu) is corresponding author. validity of a MAA to measure knee valgus during 3 functional activities (ie, single-leg drop landing, single-leg hop, and $90^{\circ}$ cut) in both healthy and ACLR populations.

\section{Methods}

\section{Participants}

Twelve participants with ACLR ( 2 males and 10 females; 24.5 [7.2] y, 164.2 [11.2] cm, 67.9 [8.2] kg) and 20 controls without ACLR (13 males and 7 females; 25.2 [2.8] y; 175.1 [7.5] cm, 72.9 [10.4] $\mathrm{kg}$ ) participated in this study. All subjects in the ACLR group had a noncontact, unilateral ACL injury with a surgical repair within the past 1 to 5 years (average 2.4 [1.4] y).

\section{Instrumentations}

A 10-camera motion analysis system (Vicon; Oxford Metrics Ltd, Oxford, UK) was used to capture lower-extremity kinematic data at $250 \mathrm{~Hz}$. For 2D analysis, video recordings of the 3 tests were captured on an iPad Air 2 tablet (Apple Inc., Cupertino, CA, USA) at 30 frames per second. The iPad was mounted on a tripod to capture frontal plane kinematics at a fixed distance of $359 \mathrm{~cm}$ from the landing zone and $35 \mathrm{~cm}$ from the floor.

\section{Procedures}

Prior to the study, subjects were given informed consent approved by the institutional review board of University of Nevada, Las Vegas. All subjects performed functional testing with simultaneous 
3D motion capture and video recording on an iPad. Reflective markers were applied by the same investigator to the lowerextremity landmarks of the participants to obtain the lowerextremity kinematics using the definition described by Stearns and Pollard. ${ }^{2}$ Each subject performed 3 functional tests in the order: single-leg drop landing, single-leg hop, and a $90^{\circ}$ cut bilaterally. During the single-leg drop landing task, participants stood on the contralateral leg, stepped forward off a $30-\mathrm{cm}$ box with the test leg, and then landed at least $30 \mathrm{~cm}$ from the box. Single-leg hop was performed with the subject being instructed to hop as far as possible. During the $90^{\circ}$ cut, participants approached the marked cutting point at the maximum speed they could confidently perform the task, beginning at $7 \mathrm{~m}$ behind the cutting point.

\section{Data Processing}

The peak knee valgus during the landing phase was obtained for each task using the 3D motion analysis and a MAA. The landing phase began at initial contact of the landing leg and ended at maximal knee flexion. If knee valgus was not observed, the minimum varus angle was obtained.

For 3D motion analysis, the reflective markers were labeled and digitized using Vicon Nexus software (Oxford Metrics Ltd), and peak knee valgus was analyzed using Visual 3D software (C-Motion, Rockville, MD). ${ }^{2}$ For $2 \mathrm{D}$ analysis, recorded videos were uploaded to Simi Move MAA (Simi Reality Motion Systems, Unterschleißheim, Germany) without further video filtering. Knee valgus was determined using the frontal plane projection angle formed by a line along the midline of the thigh to the center of the patella and a line from the center of the patella to a point bisecting the malleoli (Figure 1).,7,8 The investigator visually determined the time point of peak knee valgus for measurements.

To establish intrarater reliability, one investigator analyzed the videos on 2 separate days with at least 7 days apart. Interrater reliability was determined by comparing the measurement of the investigator to that of the other investigator.

\section{Statistical Analysis}

Values from both legs were combined for each task during analysis. Interrater and intrarater reliability were analyzed using intraclass correlation coefficients $\left(\mathrm{ICC}_{3, k}\right)$ and standard error of measurement (SEM). ICC values were classified according to the following criteria $^{5}$ : poor $(<.4)$, fair (.4-.7), good (.7-.9), and excellent (>.9). The validity was determined by comparing 2D and 3D measurements of knee valgus using a Pearson correlation coefficient. Correlation was defined as small (.1-.3), moderate (.3-.5), large $(.5-.7)$, very large (.7-.9), and extremely large (>.9). ${ }^{10}$ In addition, independent $t$ tests were used to compare knee valgus measured between 2D and 3D methods and between the 2 raters. Paired $t$ tests were used to compare the knee valgus obtained by the same rater between days. A significance level was set at .05.

\section{Results}

Our data demonstrated excellent intrarater reliability with low SEM when using a MAA to measure knee valgus in all tasks in both groups (Table 1). The interrater reliability was also excellent in all tasks for both groups (Table 1). For validity, Pearson correlation coefficients were moderate to largely correlated and significant across all tasks for healthy controls $(r=.46-.57 ; P \leq .003)$ (Figures 2A-2C) and individuals with ACLR $(r=.52-.66$;
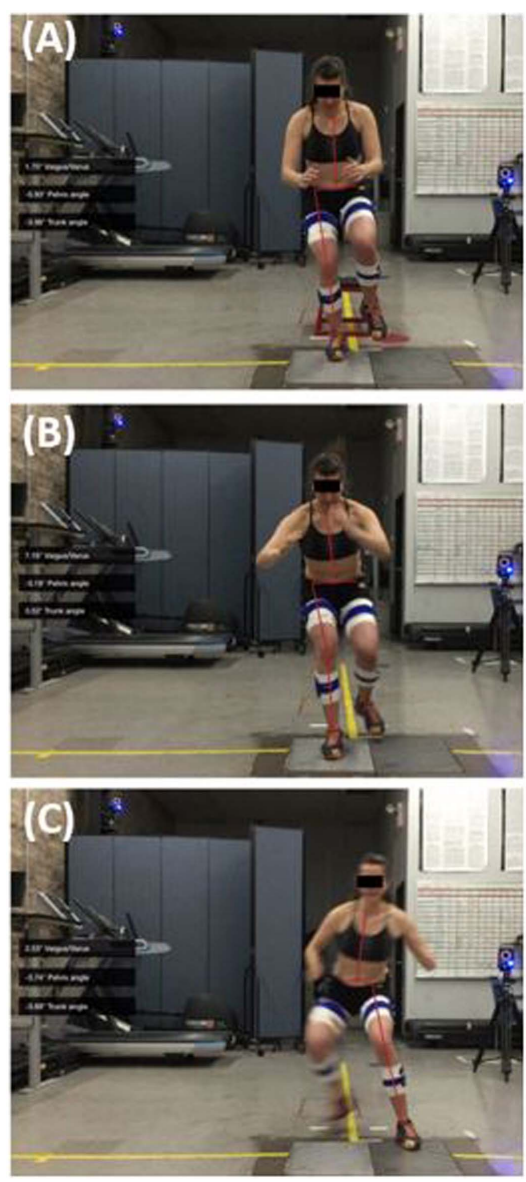

Figure 1 - Measurement of peak knee valgus angle during the landing phase using Simi Move motion analysis application during (A) single-leg drop landing, (B) single-leg hop, and (C) $90^{\circ}$ cut.

$P \leq .004$ ) (Figures 2D-2F). In addition, peak knee valgus obtained by the MAA was significantly greater than that obtained by $3 \mathrm{D}$ analysis across all tasks in both groups $(P<.001$; Table 1$)$. No differences were found between raters and within the rater for using a MAA to quantify knee valgus $(P>.05)$.

\section{Discussion}

Our data demonstrated excellent interrater and intrarater reliability for measuring knee valgus during functional activities using a MAA. However, the agreement between a 3D motion analysis system and a MAA was moderate to large and there were differences in knee valgus between the 2 methods. This indicated that the actual values obtained by a MAA may not be accurate.

The excellent intrarater and interrater reliability found in this study was similar to that reported in other studies using healthy populations. ${ }^{4,5,9}$ The interrater reliability of the existing literature ranged from moderate to excellent, with King and Belyea ${ }^{8}$ reported .45 to .99 during a drop jump task, whereas Herrington and Munro ${ }^{7}$ reported .97 to 1.0 in a single-leg landing and Mizner et al $^{9}$ reported .89 during a drop vertical jump. In terms of intrarater reliability, Herrington and Munro ${ }^{7}$ reported .58 to .96 in a single-leg landing, whereas Mizner et $\mathrm{al}^{9}$ reported .95 during drop vertical jump and Maykut et $\mathrm{al}^{4}$ reported .96 to .98 during running. Based on the 
Table 1 Repeatability (Interrater and Intrarater Reliability) of Knee Valgus Angles Measured on a MAA and the Comparisons of Knee Valgus Angle Obtained Between/Within Raters and Between MAA and 3D Methods for the Control and ACLR Groups

\begin{tabular}{|c|c|c|c|c|c|c|c|c|c|c|}
\hline \multirow[b]{3}{*}{ Single-leg drop landing } & \multicolumn{5}{|c|}{ Control } & \multicolumn{5}{|c|}{ ACLR } \\
\hline & \multicolumn{2}{|c|}{ Intrarater } & \multicolumn{2}{|c|}{ Interrater } & & \multicolumn{2}{|c|}{ Intrarater } & \multicolumn{2}{|c|}{ Interrater } & \\
\hline & $I C C$ & $S E M$, deg & $I C C$ & SEM, deg & & $I C C$ & $S E M$, deg & $I C C$ & $S E M$, deg & \\
\hline \multirow[t]{2}{*}{ Reliability } & .98 & 1.09 & .94 & 2 & & .99 & 0.6 & .98 & 0.85 & \\
\hline & Day 1 & Day 2 & Rater 1 & Rater 2 & $3 \mathrm{D}$ & Day 1 & Day 2 & Rater 1 & Rater 2 & $3 \mathrm{D}$ \\
\hline Knee valgus, deg & $5.5(8.4)$ & $5.2(7.3)$ & $5.2(7.3)$ & $6.3(8.3)$ & $-0.1(2.9)^{*}$ & $5.3(6.3)$ & $6.0(6.5)$ & $5.2(6.0)$ & $7.1(6.4)$ & $1.2(3.5)^{*}$ \\
\hline Single-leg hop & $I C C$ & $S E M$, deg & $I C C$ & $S E M$, deg & & $I C C$ & SEM, deg & $I C C$ & $S E M$, deg & \\
\hline \multirow[t]{2}{*}{ Reliability } & .98 & 1.26 & .97 & 1.64 & & .98 & 1.26 & .97 & 1.64 & \\
\hline & Day 1 & Day 2 & Rater 1 & Rater 2 & $3 \mathrm{D}$ & Day 1 & Day 2 & Rater 1 & Rater 2 & $3 \mathrm{D}$ \\
\hline Knee valgus, deg & $7.9(8.8)$ & $6.8(9.1)$ & $7.3(8.8)$ & $8.5(9.0)$ & $0.1(4.1)^{*}$ & $7.4(7.4)$ & $8.1(7.6)$ & $7.4(7.2)$ & $8.2(7.5)$ & $2.0(4.0)^{*}$ \\
\hline $90^{\circ} \mathrm{cut}$ & ICC & SEM, deg & ICC & SEM, deg & & $I C C$ & SEM, deg & ICC & SEM, deg & \\
\hline \multirow[t]{2}{*}{ Reliability } & .97 & 2.44 & .97 & 2.25 & & .97 & 2.44 & .97 & 2.25 & \\
\hline & Day 1 & Day 2 & Rater 1 & Rater 2 & 3D & Day 1 & Day 2 & Rater 1 & Rater 2 & $3 \mathrm{D}$ \\
\hline Knee valgus, deg & $12.6(14.1)$ & $13.0(13.8)$ & $12.8(13.9)$ & $15.4(13.8)$ & $7.6(4.8)^{*}$ & $17.4(10.8)$ & $18.1(10.5)$ & $17.3(10.5)$ & $19.7(11.5)$ & $8.0(5.2)^{*}$ \\
\hline
\end{tabular}

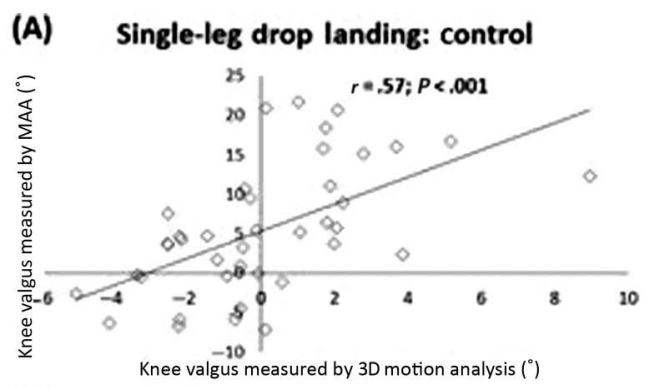

(B) Single-leg hop: control
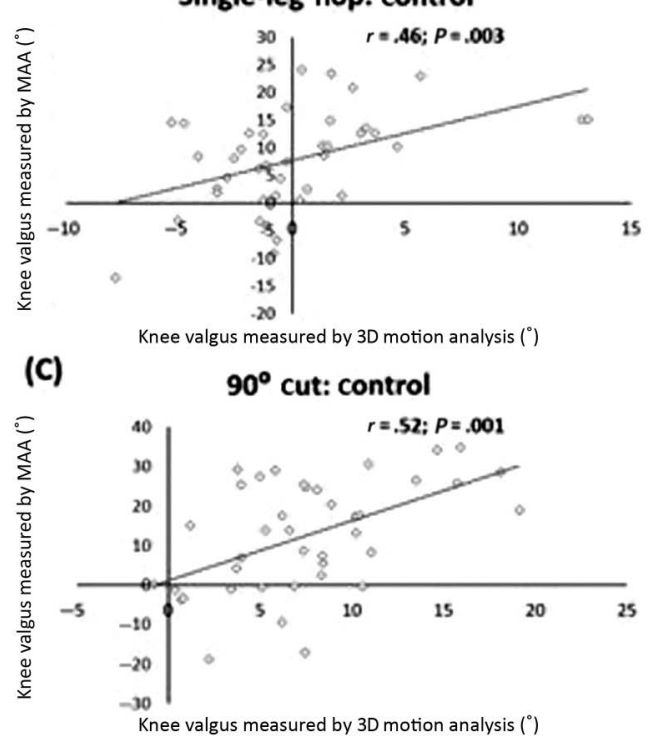

(D) Single-leg drop landing: ACLR

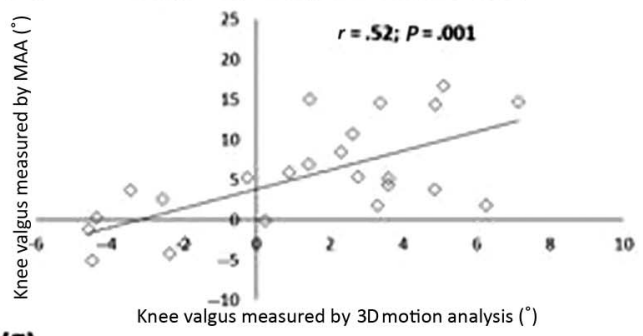

(E)
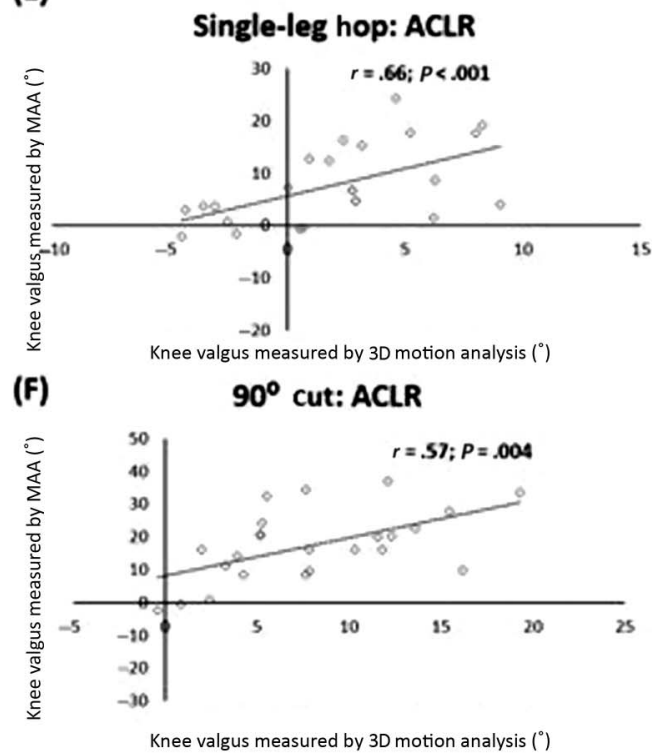

Figure 2 - The correlations between peak knee valgus angles measured by a MAA and those measured by 3D motion analysis of healthy controls during (A) single-leg drop landing, (B) single-leg hop, (C) $90^{\circ}$ cut and individuals with ACLR during (D) single-leg drop landing, (E) single-leg hop, and (F) $90^{\circ}$ cut. 3D indicates 3-dimensional; MAA, motion analysis application. 
excellent reliability of this study and its similarity to other studies that employed health controls, it can be concluded that peak knee valgus during dynamic activities can be measured reliably using a MAA for both healthy and ACLR populations.

Our study revealed a moderate to large correlation between the measurement obtained by a MAA and 3D motion analysis across single-leg drop landing, single-leg hop, and $90^{\circ}$ cut. The correlation values in our study (.46-.66) are deemed comparable to or slightly lower than those in the existing literature. Maykut et $\mathrm{al}^{4}$ found a correlation of .54 for knee valgus when comparing $2 \mathrm{D}$ to $3 \mathrm{D}$ motion analyses during running, whereas other researchers reported a correlation in single-leg landing and squatting tasks ranging from .72 to $.79 .^{3,6,7}$ As knee valgus obtained from a MAA is simply a projection angle of the result of hip adduction, hip and knee rotation, knee flexion, and knee abduction, it is thought that the differences in knee valgus measured between 2D and 3D measurements are due to the inability to account for hip/knee joint rotation and knee flexion with 2D analysis. ${ }^{4}$ In addition, our sampling rate is limited to $30 \mathrm{~Hz}$ due to hardware restriction, whereas Gwynne and Curran ${ }^{3}$ used a sampling rate of $40 \mathrm{~Hz}$ and Sorenson et $\mathrm{al}^{6}$ used a sampling rate of $240 \mathrm{~Hz}$. Another contributing factor leading to variability in validity data was the presence or absence of markers for bony landmark identification. Particularly, Gwynne and Curran ${ }^{3}$ and Munro et $\mathrm{al}^{5}$ used markers for identifying corresponding bony landmarks when making 2D measurements, which could effectively improve reliability and validity. Nevertheless, our study provides evidence regarding the validity of 2D motion analysis without the usage of additional markers, which is a common approach in clinical settings.

In conclusion, a MAA is reliable for measuring peak knee valgus in individuals with ACLR and healthy controls. However, given that the agreement between the MAA and 3D analysis was just moderate to large and there were differences in knee valgus between the 2 methods, the actual values obtained by a MAA should be viewed with caution.

\section{References}

1. Logerstedt D, Grindem H, Lynch A, et al. Single-legged hop tests as predictors of self-reported knee function after anterior cruciate ligament reconstruction: the Delaware-Oslo ACL cohort study. Am J Sports Med. 2012;40(10):2348-2356. PubMed ID: 22926749 doi:10.1177/0363546512457551

2. Stearns KM, Pollard CD. Abnormal frontal plane knee mechanics during sidestep cutting in female soccer athletes after anterior cruciate ligament reconstruction and return to sport. Am J Sports Med. 2013;41(4):918-923. PubMed ID: 23425687 doi:10.1177/ 0363546513476853

3. Gwynne CR, Curran SA. Quantifying frontal plane knee motion during single limb squats: reliability and validity of 2-dimensional measures. Int J Sports Phys Ther. 2014;9(7):898-906. PubMed ID: 25540705

4. Maykut JN, Taylor-Haas JA, Paterno MV, DiCesare CA, Ford KR. Concurrent validity and reliability of 2D kinematic analysis of frontal plane motion during running. Int J Sports Phys Ther. 2015;10(2): 136-146. PubMed ID: 25883862

5. Munro A, Herrington L, Carolan M. Reliability of 2-dimensional video assessment of frontal-plane dynamic knee valgus during common athletic screening tasks. J Sport Rehabil. 2012;21(1):7-11. PubMed ID: 22104115 doi:10.1123/jsr.21.1.7

6. Sorenson B, Kernozek TW, Willson JD, Ragan R, Hove J. Two- and three-dimensional relationships between knee and hip kinematic motion analysis: single-leg drop-jump landings. J Sport Rehabil. 2015;24(4):363-372. PubMed ID: 25658442 doi:10.1123/jsr.20140206

7. Herrington L, Munro A. Drop jump landing knee valgus angle; normative data in a physically active population. Phys Ther Sport. 2010;11(2):56-59. PubMed ID: 20381002 doi:10.1016/j.ptsp.2009. 11.004

8. King DL, Belyea BC. Reliability of using a handheld tablet and application to measure lower-extremity alignment angles. J Sport Rehabil. 2015;T24(4).

9. Mizner RL, Chmielewski TL, Toepke JJ, Tofte KB. Comparison of 2-dimensional measurement techniques for predicting knee angle and moment during a drop vertical jump. Clin J Sport Med. 2012; 22(3):221-227. PubMed ID: 22544058 doi:10.1097/JSM.0b013 e31823a46ce

10. Hopkins WG, Marshall SW, Batterham AM, Hanin J. Progressive statistics for studies in sports medicine and exercise science. Med Sci Sports Exerc. 2009;41(1):3-13. PubMed ID: 19092709 doi:10.1249/ MSS.0b013e31818cb278 